\title{
Spinogenesis and Pruning Scales across Functional Hierarchies
}

\author{
Guy N. Elston, ${ }^{1}$ Tomofumi Oga (小賀智文) ${ }^{2}$ and Ichiro Fujita (藤田一郎) ${ }^{2}$ \\ ${ }^{1}$ Centre for Cognitive Neuroscience, Sunshine Coast, Queensland 4562, Australia, and ${ }^{2}$ Graduate School of Frontier Biosciences, Osaka University, \\ Toyonaka, Osaka 560-8531, Japan
}

Spinogenesis and synaptic pruning during development are widely believed to subserve connectional specificity in the mature CNS via Hebbian-type reinforcement. Refinement of neuronal circuit through this "use it or lose it" principle is considered critical for brain development. Here we demonstrate that the magnitude of spinogenesis and pruning in the basal dendritic trees of pyramidal cells differ dramatically among sensory, association, and executive cortex. Moreover, somewhat counterintuitively, we demonstrate that the dendritic trees of pyramidal cells in the primary visual area actually lose more spines than they grow following the onset of visual experience. The present findings reveal that the process of synaptic refinement differs not only according to time, but also location.

\section{Introduction}

The structure of neuronal dendritic trees is markedly specialized among different cortical areas in the adult brain (Jacobs and Scheibel, 2002; Elston, 2003). The dendritic trees of pyramidal cells, the most abundant neuronal type in the cerebral cortex (DeFelipe and Fariñas, 1992), vary in their size, branching complexity, and number of putative excitatory inputs ("spines") among cortical areas. These differences are systematic such that the dendritic trees of pyramidal cells become increasingly more branched and spiny with progression from primary sensory to association and "executive" or prefrontal cortex. These specializations in neuron structure are believed to endow different biophysical properties and patterns of connectivity, thus providing specialized substrates for different behavioral complexities (Jacobs and Scheibel, 2002; Chklovskii et al., 2004; Elston, 2007; Spruston, 2008).

Presently available data in the primate suggest that there may be different temporal profiles for spinogenesis and pruning in different regions of the cerebral cortex (Huttenlocher and Dabholkar, 1997), and that these profiles may vary among species (Rakic et al., 1986). These findings have been fleshed out by either quantifying the density of synapses in the neuropil, or dendritic spines along labeled dendrites. However, there are no quantitative empirical data available regarding the number of synapses formed within the dendritic trees of populations of neurons of specified type among cortical areas. Here we performed a systematic study of the basal dendritic trees of pyramidal cells in sensory,

Received 0ct. 28, 2008; revised Dec. 16, 2008; accepted Jan. 14, 2009

This work was supported by grants from the Japan Science and Technology Agency (Core Research for Evolutional Science and Technology), the Ministry of Education, Culture, Sports, Science, and Technology (17022025), Osaka University Global Centers of Excellence Program, and the Hear and Say Centre, Australia. We thank Tsuguhisa Okamoto for help in preparation of this manuscript.

Correspondence should be addressed to Dr. Guy N. Elston, Centre for Cognitive Neuroscience, 60 Duke Road, Doonan, Sunshine Coast, Queensland 4562, Australia. E-mail: guyelston@yahoo.com.

D01:10.1523/JNEUROSCI.5216-08.2009

Copyright $\odot 2009$ Society for Neuroscience $\quad$ 0270-6474/09/293271-05\$15.00/0 association, and executive cortex to compare the magnitude of spinogenesis and pruning during development.

\section{Materials and Methods}

Macaque monkeys (Macaca fascicularis) aged from $2 \mathrm{~d}$ old (2D), through 3 weeks, 3.5 months $(3.5 \mathrm{M}), 7 \mathrm{M}, 18 \mathrm{M}$, and $28 \mathrm{M}$ to 4.5 years old were included for analyses. We used one animal for each age (Table 1). These time points were chosen according to the following rationale: $2 \mathrm{D}$ postnatal was the youngest animal we could obtain, 3 weeks of age correlates with the window of the critical period of ocular dominance shift according to Horton and Hocking (1997), 3.5 months of age corresponds to the peak of spine density according to Rakic et al. (1986) and the end of the critical period according to Horton and Hocking (1997), 7 months of age corresponds to double this time, and 4.5 years corresponds to young adulthood.

The animals were sedated with ketamine hydrochloride (Ketalar, 25 $\mathrm{mg} / \mathrm{kg}$ i.m., Sankyo) and overdosed with sodium pentobarbital (Nembutal, $75 \mathrm{mg} / \mathrm{kg}$ i.v. or i.p., Dainippon Sumitomo Pharma) and perfused intracardially with $0.1 \mathrm{M}$ potassium phosphate buffer saline, $\mathrm{pH} 7.2$, followed by $4 \%$ paraformaldehyde (Merck). Blocks of tissue were excised from the superior pole of the exposed occipital operculum, corresponding to the central $5-8^{\circ}$ in the visual field representation in the primary visual cortex (V1) (Daniel and Whitteridge, 1961), the inferior temporal gyrus anterior to the posterior middle temporal sulcus, corresponding to area TEpd of Yukie et al. (1990) (see also Seltzer and Pandya, 1978), and the inferior portion of the arcuate gyrus in the prefrontal cortex (PFC), corresponding to area 12vl of Preuss and Goldman-Rakic (1991). The blocks were trimmed and flattened, and then sliced parallel to the cortical surface at $250 \mu \mathrm{m}$ thick (Fig. $1 \mathrm{~A}$ ). To avoid possible confounds from gender or hemispheric differences in the morphology of pyramidal cells, we used only the right hemisphere of male animals. Slices were incubated in 4,6-diamidino-2-phenylindole (DAPI; Sigma-Aldrich) solution for visualizing somata (Fig. $1 B$ ).

Pyramidal cells were individually injected with $8 \%$ Lucifer Yellow (Sigma-Aldrich) under visual guidance by UV illumination (Fig. 1C). All cell bodies of injected cells were located in supragranular layer, immediately superior to the granular layer, which can easily be identified in DAPI preparations (see Fig. 3 of Elston and Rosa, 1997). Thus, only cells located in layers IIIB and IIIC according to the terminology of Hassler (1966) were included here. The region within each cortical area in which we 
Table 1. The number of cells analyzed for each cortical area/age group

\begin{tabular}{|c|c|c|c|c|c|c|c|}
\hline & $2 D$ & $3 W$ & $3.5 \mathrm{M}$ & $7 \mathrm{M}$ & $18 \mathrm{M}$ & $28 \mathrm{M}$ & $4.5 Y$ \\
\hline Animal & $\mathrm{Cl} 9$ & Cl1 & Cl10 & $\mathrm{Cl} 8$ & DM4 & RM12 & MF1 \\
\hline BW & 0.35 & 0.56 & 0.56 & 0.70 & - & 1.7 & - \\
\hline V1 & 25 & 41 & 29 & 34 & 0 & 135 & 22 \\
\hline TE & 23 & 38 & 27 & 52 & 21 & 0 & 31 \\
\hline PFC & 37 & 33 & 32 & 54 & 0 & 0 & 20 \\
\hline
\end{tabular}

D, W, M, Y, Postnatal days, weeks, months, and years, respectively; BW, body weight $(\mathrm{kg}) ;-$, not measured. Total number of cells, 654 .

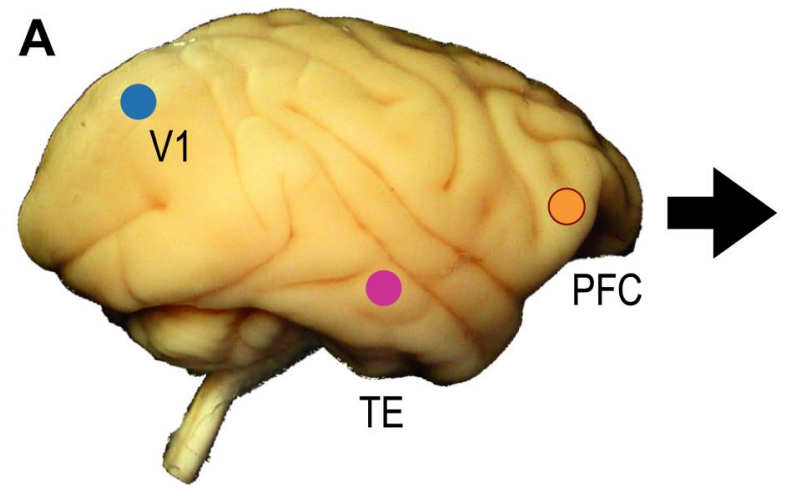

Trim \& Flatten

Slice
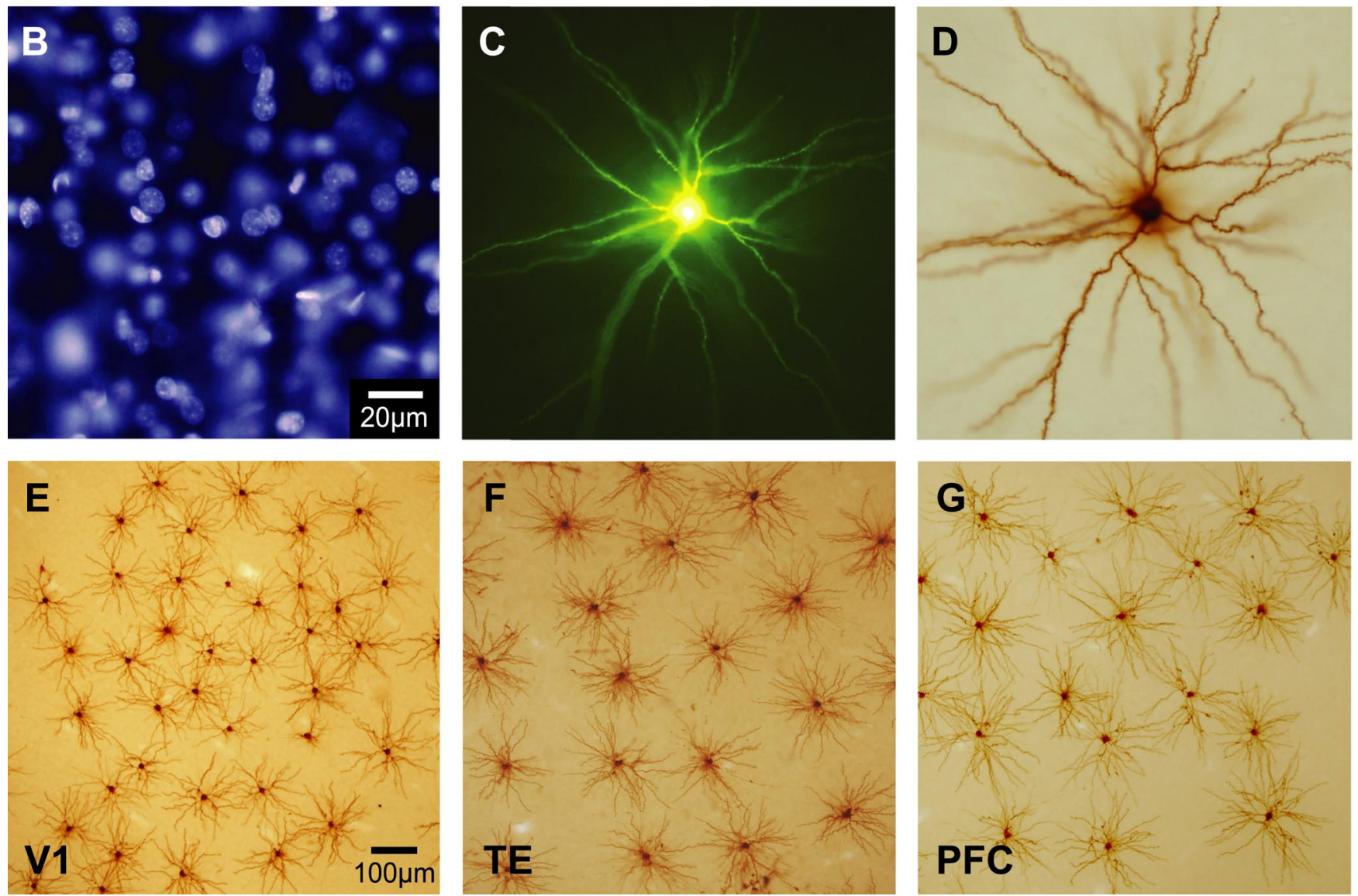

Figure 1. Experimental procedures and labeled cells. The animals were lightly fixed with paraformaldehyde, and the brain was removed, trimmed and flattened, and sliced at $250 \mu \mathrm{m}$ tangentially to the cortical surface $(\boldsymbol{A})$. Slices were incubated in DAPI solution to visualize nuclei of neurons $(\boldsymbol{B})$. Layer 3 pyramidal cells were individually injected with Lucifer yellow $(\boldsymbol{C})$ and reacted for DAB product (D). Examples of injected cells from V1, TE, and PFC of a 3-week-old monkey are shown in $\boldsymbol{E}$, $\boldsymbol{F}$, and $\mathbf{G}$, respectively. Scale bars: $20 \mu \mathrm{m}(\boldsymbol{B}-\boldsymbol{D}), 100 \mu \mathrm{m}(\boldsymbol{E}-\boldsymbol{G})$.

injected cells was determined by their relative location to gross anatomical landmarks in the tangential plane. Interanimal consistency in sampling within each cortical area was ensured by triangulating across gross landmarks in brains of different sizes.

The sections were processed for a biotinylated antibody (Invitrogen) raised against Lucifer Yellow at a concentration of 1:5000 in stock solu- tion (2\% bovine serum albumin, $1 \%$ Triton X-100, and $5 \%$ sucrose in 0.1 $\mathrm{M}$ phosphate buffer). They were then processed with a biotin-horseradish peroxidase complex (Amersham Biosciences; 1:100 in 0.1 M phosphate buffer) and reacted for a light stable diaminobenzidine (DAB; Sigma-Aldrich) reaction product (Fig. $1 D$ ). Figure $1 E-G$ shows examples of injected cells in V1, TE, and PFC of a 3-week-old monkey. 

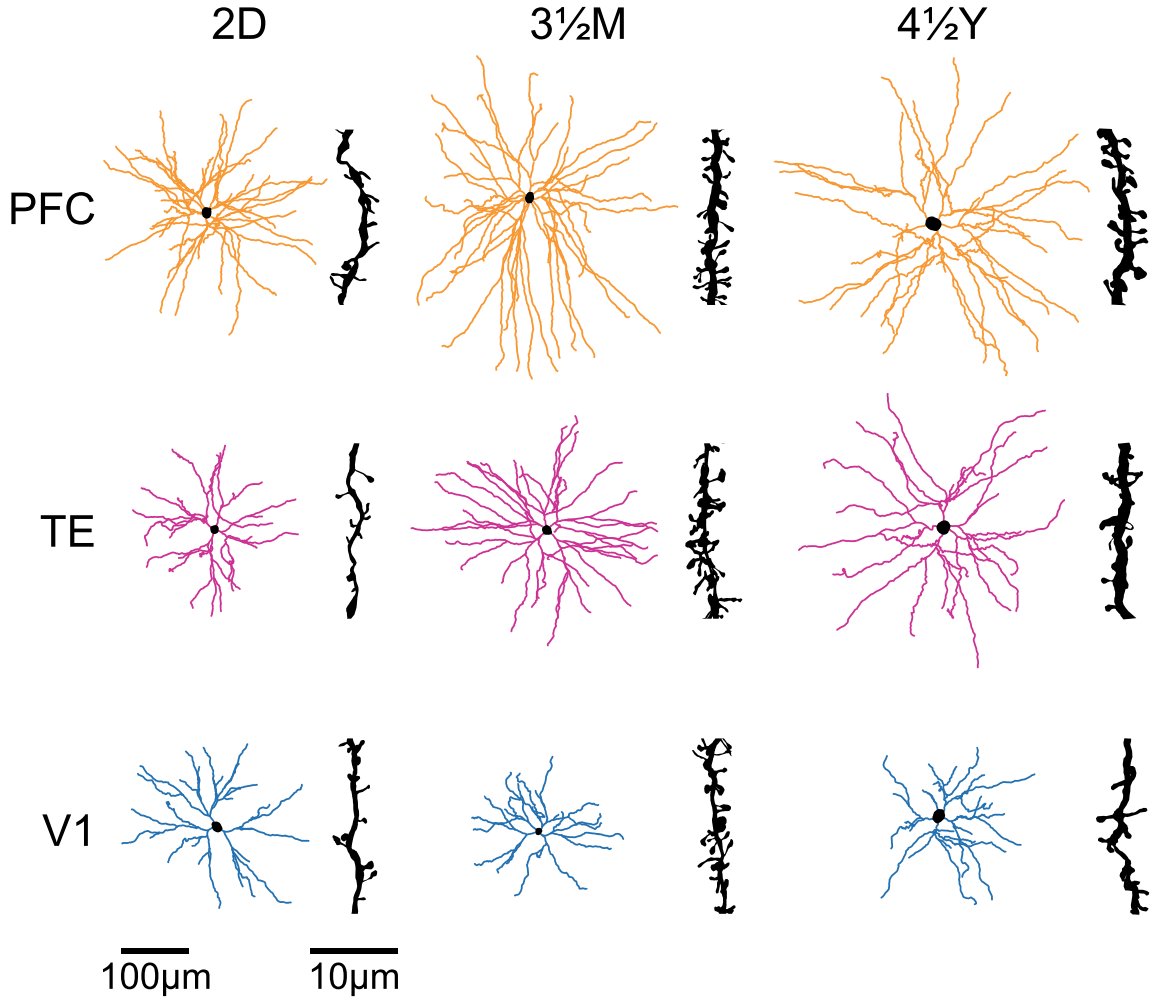

Figure 2. Skeletonized reconstructions of the basal dendritic trees of pyramidal cells (in the tangential plane) sampled from the primary visual (V1), inferotemporal visual association (TE), and granular prefrontal (PFC) cortex at $2 \mathrm{~d}$ postnatal (2D), 3.5 months postnatal $(31 / 2 \mathrm{M})$, and 4.5 years postnatal $(41 / 2 \mathrm{Y})$. The illustrated cells represent the average of all cells sampled for each cortical area/age group. Insets illustrate the spine density for cells of each cortical area/age group at $50-70 \mu \mathrm{m}$ from the cell body.

The basal dendritic trees of 654 cells from V1, TE, and PFC were reconstructed in three dimensions with a computer-aided tracing system (Neurolucida; MBF Bioscience) to determine their branching structure. Only the basal dendrites issuing from the cell body were included for analyses. Approximately 20-50 cells were included for analyses in each of V1, TE, and PFC of each animal (see Table 1 for the number of cells analyzed for each age/area group). Over 65,000 spines were drawn with a camera lucida system at $100 \times$ magnification (numerical aperture of objective lens and condenser, 1.40), and quantified as a function of distance from the cell body to the distal tips of the dendrites. We selected horizontally projecting dendrites for our calculations of spine densities to avoid trigonometric error. Spine densities were calculated per $10 \mu \mathrm{m}$ interval along the entire length of 20 individually drawn, randomly selected, dendrites in each cortical area for each age group. Three dimensional reconstructions were reduced to two dimensions for these analyses so the data could be compared directly with previous papers by one of the authors (G.N.E.). All experiments were performed according to the National Institutes of Health guidelines for the Care and Use of Laboratory Animals and were approved by the Animal Experiment Committee of Osaka University.

\section{Results}

In all three cortical regions we found a successive increase in spine density with increasing age before reaching a peak and declining to the adult values (Figs. 2, 3A). In agreement with previous reports relating to synapse density in the cerebral cortex (Rakic et al., 1986), we found that spinogenesis in the basal dendrites of pyramidal cells was greatest at 3.5 months of age in the different cortical areas. However, while spine density along the dendrites increased at a similar rate up to 3.5 months of age, thereafter it decreased at different rates among V1, TE, and PFC (two-way ANOVA, $p<0.0001$ for age, area, and interaction; $F_{(4)}=584, F_{(2)}=139, F_{(8)}=36.0$ for age,
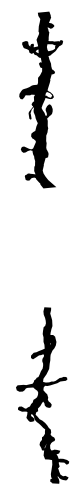

area, and interaction, respectively). Specifically, there was a successive increase in spine loss through PFC, TE, and V1. Similar changes occurred both in the proximal and distal parts of the basal dendrites (Fig. 3A).

To further examine rates of spinogenesis and pruning within the basal dendritic trees of individual pyramidal cells, as opposed to within the cortical depth, we summed the product of the average spine density as a function of distance from the cell body by the number of branches per corresponding Sholl annulus over the entire basal dendritic tree of intracellularly injected neurons. By doing so we were able to calculate an estimate of the total number of spines in the basal dendritic tree of the average neuron in each cortical region (see Elston, 2001). These analyses revealed that layer 3 pyramidal cells in PFC grew considerably more spines $(15,900)$ in their basal dendritic trees than did those in TE $(10,400)$ and V1 (3900) (Fig. 3B). Thereafter, cells in these three regions pruned different numbers of spines (7400 in PFC, 4200 in TE, and 3000 in V1) before stabilizing to adult values of 8500,6200 , and 900 spines, respectively. Somewhat surprisingly, these calculations reveal that following postnatal visual onset more spines are pruned from the basal dendritic trees of pyramidal cells in $\mathrm{V} 1$ than are grown. In TE and PFC, however, the basal dendritic trees of pyramidal cells in the mature brain had almost double the number of spines as those observed in the newborn brain. Interestingly, the number of spines $(>5000)$ pruned from the dendritic trees of pyramidal cells in the PFC between 3.5 months of age and 4.5 years of age, corresponds to $>8$ times the total number of spines found in the dendritic trees of pyramidal cells in $\mathrm{V} 1$ of the mature brain.

\section{Discussion}

Here we demonstrate that there are marked differences in the number of spines grown within the basal dendritic trees of individually identified pyramidal cells among V1, TE, and PFC. Moreover, pruning of spines from pyramidal cell dendrites also differs among these cortical areas. Regional differences in the number of dendritic spines (putative excitatory inputs) in the basal dendrites of pyramidal cells reported during spinogenesis and pruning, and in the adult, provide further evidence in support of the theory relating neuronal complexity and functional hierarchies in the brain [for review, see Jacobs and Scheibel (2002), Elston (2007), and Spruston (2008)]. It remains to be determined how genetic and epigenetic mechanisms interact to produce such regional specialization in the brain. It is worth noting that even in the newborn brain cells in PFC are $>2$ times more spinous than those in V1.

The present observations in V1 are somewhat counterintuitive to classical Hebbian type reinforcement. Pyramidal cells in V1 lose more spines than they grow following the onset of visual experience. This finding is all the more curious as 
normal visual experience is required for normal spinogenesis in pyramidal cells in V1 (Valverde, 1967). Thus it appears as though the onset of visual experience promotes exuberant spinogenesis, but an even larger number of spines are lost during maturation. Would this observation also be true for TE and PFC we might conclude that many of these spines simply did not form synapses [for review, see Kasai et al. (2003) and Bourne and Harris (2007)]. However, the present data for peak spine density at 3.5 months of age correspond with previous reports for peak synapse density (Rakic et al., 1986) in all three cortical regions. Electron microscope studies will provide further insights as to why we found different developmental trends between V1 and TE/PFC.

The present data, coupled with the results of electrophysiological studies of pyramidal cells (Murayama et al., 1997) suggest that the efficacy of Hebbian-type synaptic reinforcement differs not only according to time, but also location within the brain. Given the regional differences in the magnitude of spinogenesis and pruning among cortical areas, the differences in the time course of spinogenesis and pruning among species (Rakic et al., 1986; Huttenlocher and Dabholkar, 1997), and regional specialization in pyramidal cell structure observed in the adult primate brain (Elston and Rosa, 1997; Jacobs et al., 1997, 2001; Elston, 2000), it is becoming increasingly more parsimonious that synaptic activity thresholds that reinforce synapses and stabilize dendritic spines may vary across cortex. Thus, depending on how and where, even if you use it you still may lose it.
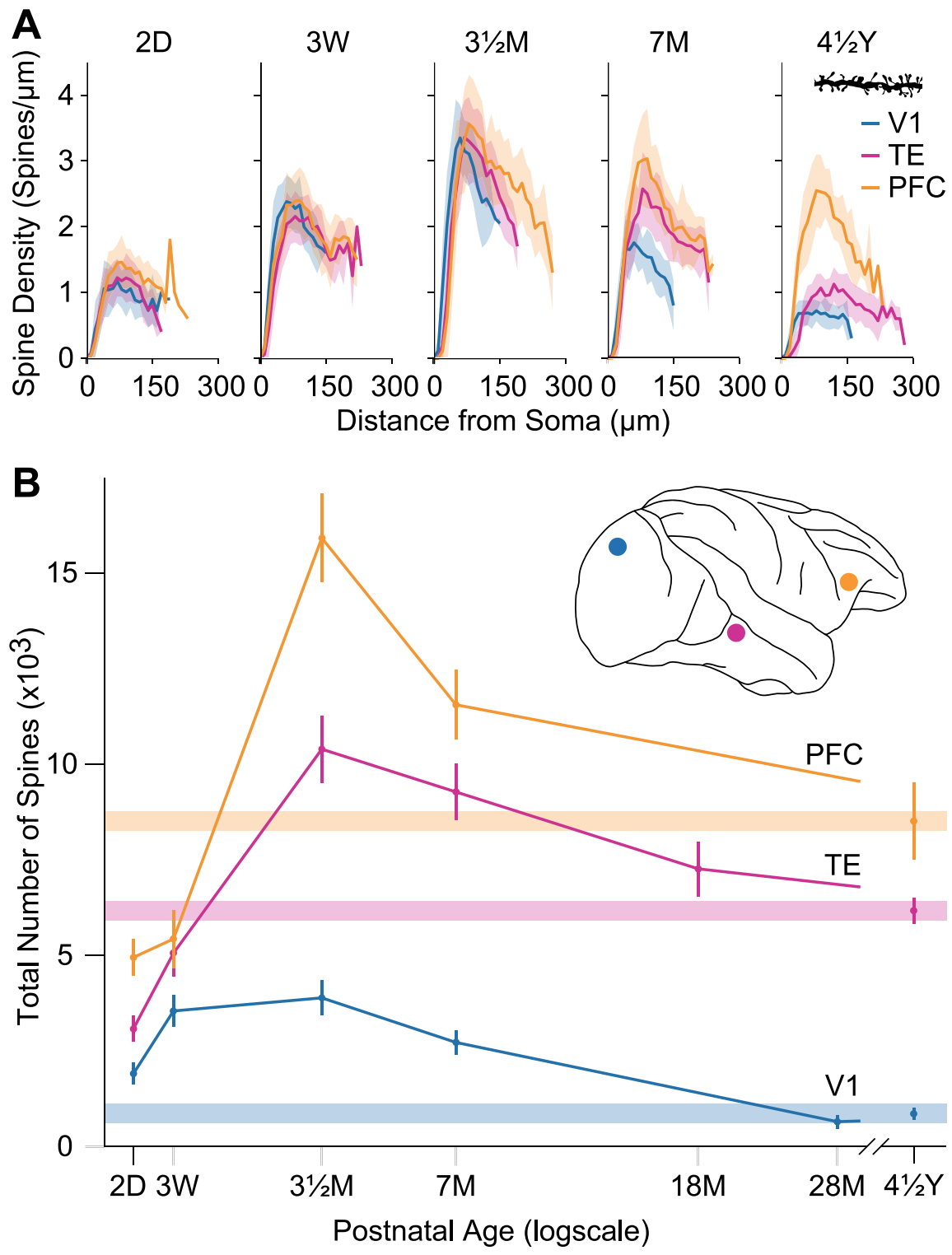

Figure 3. Plots of the spine density, as calculated per $10 \mu \mathrm{m}$ of dendrite from the cell body to the distal tips of the dendrites, of pyramidal cells in V1, area TE, and PFC at five different postnatal ages. Thick lines and shaded areas indicate the means and SDs, respectively $(\boldsymbol{A})$. Total number of dendritic spines in the basal dendritic tree of the "average" cell in each cortical area/age group. Inset indicates the location of sampled areas. Error bars indicate SDs $(\boldsymbol{B})$.

\section{References}

Bourne J, Harris KM (2007) Do thin spines learn to be mushroom spines that remember? Curr Opin Neurobiol 17:381-386.

Chklovskii DB, Mel BW, Svoboda K (2004) Cortical rewiring and information storage. Nature 431:782-788.

Daniel PM, Whitteridge D (1961) The representation of the visual field on the cerebral cortex in monkeys. J Physiol 159:203-221.

DeFelipe J, Fariñas I (1992) The pyramidal neuron of the cerebral cortex: morphological and chemical characteristics of the synaptic inputs. Prog Neurobiol 39:563-607.

Elston GN (2000) Pyramidal cells of the frontal lobe: all the more spinous to think with. J Neurosci 20:RC95(1-4).

Elston GN (2001) Interlaminar differences in the pyramidal cell phenotype in cortical areas $7 \mathrm{~m}$ and STP (the superior temporal polysensory area) of the macaque monkey. Exp Brain Res 138:141-152.

Elston GN (2003) Cortex, cognition and the cell: new insights into the pyramidal neuron and prefrontal function. Cereb Cortex 13:1124-1138.

Elston GN (2007) Specializations in pyramidal cell structure during primate evolution. In: Evolution of nervous systems (Kaas JH, Preuss TM, eds), pp 191-242. Oxford: Academic.
Elston GN, Rosa MGP (1997) The occipitoparietal pathway of the macaque monkey: comparison of pyramidal cell morphology in layer III of functionally related cortical visual areas. Cereb Cortex $7: 432-452$.

Hassler R (1966) Comparative anatomy of the central visual system in dayand night-active primates. In: Evolution of the forebrain (Hassler R, Stephen $\mathrm{H}$, eds), pp 419-434. Stuttgart: Thieme.

Horton JC, Hocking DR (1997) Timing of the critical period for plasticity of ocular dominance columns in macaque striate cortex. J Neurosci 17:3684-3709.

Huttenlocher PR, Dabholkar AS (1997) Regional differences in synaptogenesis in human cerebral cortex. J Comp Neurol 387:167-178.

Jacobs B, Scheibel AB (2002) Unity and diversity. In: Cortical areas (Schüz A, Miller R, eds), pp 111-131. London: Taylor and Francis.

Jacobs B, Driscoll L, Schall M (1997) Life-span dendritic and spine changes in areas 10 and 18 of human cortex: a quantitative Golgi study. J Comp Neurol 386:661-680.

Jacobs B, Schall M, Prather M, Kapler E, Driscoll L, Baca S, Jacobs J, Ford K, Wainwright M, Treml M (2001) Regional dendritic and spine 
variation in human cerebral cortex: a quantitative Golgi study. Cereb Cortex 11:558-571.

Kasai H, Matsuzaki M, Noguchi J, Yasumatsu N, Nakahara H (2003) Structure-stability-function relationships of dendritic spines. Trends Neurosci 26:360-368.

Murayama Y, Fujita I, Kato M (1997) Contrasting forms of synaptic plasticity in monkey inferotemporal and primary visual cortices. Neuroreport 8:1503-1508.

Preuss TM, Goldman-Rakic PS (1991) Myelo- and cytoarchitecture of the granular frontal cortex and surrounding regions in the strepsirhine primate Galago and the anthropoid primate Macaca. J Comp Neurol 310:429-474.

Rakic P, Bourgeois JP, Eckenhoff MF, Zecevic N, Goldman-Rakic PS (1986)
Concurrent overproduction of synapses in diverse regions of the primate cerebral cortex. Science 232:232-235.

Seltzer B, Pandya DN (1978) Afferent cortical connections and architectonics of the superior temporal sulcus and surrounding cortex in the rhesus monkey. Brain Res 149:1-24.

Spruston N (2008) Pyramidal neurons: dendritic structure and synaptic integration. Nat Rev Neurosci 9:206-221.

Valverde F (1967) Apical dendritic spines of the visual cortex and light deprivation in the mouse. Exp Brain Res 3:337-352.

Yukie M, Takeuchi H, Hasegawa Y, Iwai E (1990) Differential connectivity of inferotemporal area TE with the amygdala and the hippocampus in the monkey. In: Vision, memory, and the temporal lobe (Iwai E, Mishkin M, eds), pp 129-135. New York: Elsevier. 